\title{
Repertorio de artistas mujeres en la historiografía canónica del arte argentino: un panorama de encuentros y desencuentros
}

\author{
Hib, Ana
}

Resumen:

El presente texto tiene como objetivo trazar un mapa de las menciones de aquellas artistas que atraviesan nuestra historiografía del arte desde sus comienzos hasta fines de 1930 , comparando textos más cercanos en el tiempo para poder revisar cambios y permanencias. En nuestros inicios, las mujeres se instruyen, crean, y aparecen en críticas periodísticas, pero el reconocimiento de los textos canónicos o de los premios no llega o se encuentra de manera reducida. A partir de la década de 1940 y hasta la actualidad, la cantidad y el registro de las artistas mujeres se multiplica, y se suman agentes como historiadoras del arte, críticas, curadoras, galeristas, coleccionistas, gestoras, directoras de museos y de centros culturales.

\section{Cuadernos del Centro de Estudios de} Diseño y Comunicación Nº 60

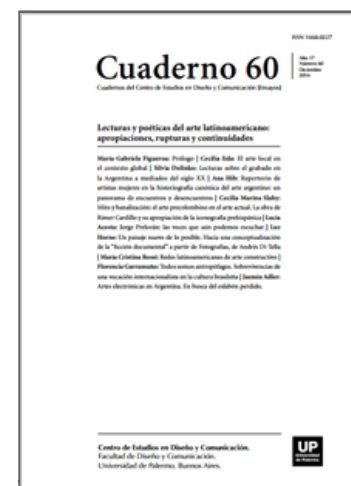

ISSN: 1668-0227

Lecturas y poéticas

del arte

latinoamericano:

apropiaciones,

rupturas y

continuidades

Año XVII, Diciembre 2016, Buenos Aires,

Argentina | 176 páginas

descargar PDF ver índice de la publicación

Ver todos los libros de la publicación

compartir en Facebook

Palabras clave:

arte argentino - artistas mujeres - historiografía del arte - canon.

$\left(^{*}\right)$ Licenciada y Profesora en Artes (UBA), docente en la cátedra de Psicología del Arte (FFyL, UBA), investigadora independiente especializada en conservación y gestión de archivos de arte, y editora.

El presente texto tiene un objetivo muy concreto: el de trazar un mapa de las menciones de artistas argentinas (y en algunos casos extranjeras radicadas en nuestro país) de hasta fines de la década del 30, que atraviesan nuestra historiografía del arte desde sus inicios, realizando algunas comparaciones con textos más cercanos en el tiempo e incluso contemporáneos para poder revisar las permanencias y los cambios ocurridos. 
¿A qué nos lleva el conocer estos registros? No por cierto a que permanezcan como meras nóminas o descripciones cuantitativas. Para una interpretación de estas menciones (y omisiones) resulta insoslayable leer Omisiones y presencias veladas: artistas argentinas en las historias del arte, de Georgina Gluzman (2010); allí, gracias al detallado estudio de bibliografía del arte feminista realizado por la autora, veremos a qué tradición responden estos relatos canónicos (queda muy claro que a la vasariana), observaremos de qué modo las artistas son destacadas (como hija de, esposa de, amante de, pintora de tal o cual personaje - varón eminente), de qué manera es descripto su estilo, qué rasgos lo caracterizan (ternura, sutileza, delicadeza, belleza, elegancia, gracia, suavidad, fina sensibilidad; salvo en el caso de la "viril e impetuosa" Raquel Forner). También es ineludible la precursora mirada de Laura Malosetti Costa para las VI Jornadas de Historia de las Mujeres (Malosetti Costa, 2000), y del año siguiente, su libro Los primeros modernos (Malosetti Costa, 2001).

Se puede hablar de una "fortuna bibliográfica" de aquellas creadoras que sí ingresaron en "La" Historia del Arte; de forma acotada si se compara en número con los artistas varones, mientras sabemos, gracias a estudios como los de Gluzman y Malosetti y a incursiones en distintos archivos o repositorios1, que en el mismo período las mujeres eran muy numerosas como cursantes de estudios de pintura y dibujo, aunque en menor medida en grabado y escultura, y se presentaban a Salones también en forma frecuente y en cantidad importante2.

Es así que las mujeres se instruyen, crean, participan de forma activa en eventos relevantes dentro del campo artístico y se las registra en críticas periodísticas, pero el reconocimiento "oficial" de la historiografía canónica o de los premios no llega o se encuentra de manera muy reducida con respecto a sus accionares.

Los textos canónicos

El enfoque hegemónico de la historiografía tradicional argentina, de modo similar a la europea, presenta una realidad de grandes nombres de artistas varones consagrados y, si es que se ocupa de nombrar a las mujeres, lo hace de manera escueta, general y en muchos casos, considerándolas como excepciones.

Los textos que han sido analizados para este relevamiento son: - Schiaffino, Eduardo (1933). La pintura y la escultura en Argentina. Buenos Aires: edición del autor.

- Pagano, José León (1937-1940). El arte de los argentinos. Buenos Aires: edición del autor, 3 tomos (asimismo, se consultó la edición en un solo tomo con la revisión que J. L. Pagano (h) hace casi medio siglo más tarde: El arte de los argentinos, Buenos Aires, Editorial y Librería Goncourt, 1981).

- Romero Brest, Jorge (1951). Pintores y grabadores rioplatenses. Buenos Aires: Argos.

- Payró, Julio E. (1962). 23 pintores de la Argentina. 1810-1900. Buenos Aires: Eudeba.

- Córdova Iturburu, Cayetano (1958). La pintura argentina del siglo XX. Buenos Aires: Atlántida.

- Córdova Iturburu, Cayetano (1978). 80 años de pintura argentina. Del pre-impresionismo a la novísima figuración. Buenos Aires: Librería de la Ciudad.

Podemos decir que, a pesar de la presencia de relatos anteriores, existe un cierto consenso acerca del papel pionero ocupado por Eduardo Schiaffino en el proceso de formación de la disciplina. En su obra, es relevante 
señalar que la mención de las mujeres es sumamente escasa, lo cual da origen a una larga serie de relatos donde ellas se encuentran en posiciones secundarias. En cambio, José León Pagano da cuenta de muchas artistas, e incluso en el Tomo III de su emblemático libro El arte de los argentinos presenta un capítulo titulado "La contribución femenina", donde la estrategia de la separación por un lado las destaca y llama la atención sobre ellas, al tiempo que produce un movimiento de segregación3.

Es en este caso en que se observa la mayor presencia de artistas mujeres comparada con los relatos de otras historias del arte canónicas, y si bien advertimos la inserción de sus nombres y el reconocimiento de sus obras en ítems o capítulos exclusivos, y analizando el texto notamos marcas discursivas comunes a la época, tenemos que reconocer que Pagano fue el único historiador que se ocupó de mantener vivas las historias de estas artistas. El Archivo José León Pagano, situado en el Museo de Arte Moderno de Buenos Aires, refleja de manera mucho más contundente este interés del artista y crítico: se encontraron allí más de 1.500 documentos vinculados con ellas; un reservorio documental único por la calidad de los materiales, que incluye fotografías de obras perdidas, catálogos, recortes de sus críticas en La Nación y correspondencia. Alrededor de 230 artistas han sido registradas en su archivo, mientras 23 se encuentran analizadas en El arte de los argentinos (a lo que se suma la mención de otras tantas), como veremos más adelante.

Posteriormente, en las obras de quienes continuaron con la tarea de construir historias generales del arte argentino bajo la égida de la Modernidad, como Cayetano Córdova Iturburu, Julio E. Payró y Jorge Romero Brest, la presencia de las mujeres no estará mucho más destacada, o directamente será nula.

A continuación, realizaremos un punteo por cada título del corpus seleccionado.

- E. Schiaffino (1933): Solo figura Procesa Sarmiento de Lenoir.

- J. L. Pagano (1937-1940); con distintos niveles de desarrollo, se encuentran: Tomo I (1937): Leónie Matthis (solo nombra y reproduce dos obras), Adrienne Pauline Bacle, Luisa Sánchez de Arteaga, Procesa Sarmiento de Lenoir, Eugenia Belín Sarmiento, Daría Echagüe de Santibáñez, María Obligado de Soto y Calvo, Julia Wernicke (con textos e imágenes de obras).

Tomo III (1940), en el capítulo "La contribución femenina": Lía Gismondi, Catalina Mórtola de Bianchi (en su apartado se enlistan otras grabadoras: María Catalina Otero Lamas, Elba Villafañe, Margarita Portela Lagos, Hemilce Saforcada, María Luisa García Iglesias, María Teresa Valeiras de Gigli, Mané Bernardo, María Manuela Alles Monasterio, Leónidas Maggiolo, María Esther Leanes, Clara Carrié, Delia Teresa Copello, Eugenia Badaracco, Carmen Souza Brazuna, Eglantina Amelia Villagra, Lola Chevalier, Aída Vaisman, María Rocchi, Sor María Verónica, Sor María Cristina), Ana Weiss de Rossi, Aurora de Pietro de Torras, Lola Mora, Lía Correa Morales de Yrurtia, Ángela Adela Vezzeti, Raquel Forner, María Escudero, Emilia Bertolé, María Mercedes Rodrigué de Soto Acebal, Carlota Stein, Norah Borges de Torre, Biyina Klappenbach, Dora Cifone, Hildara Pérez de Llanso, Andreína Bai de De Luca, todas con textos y reproducciones de obra; en pintura se anexa un listado de nombres (sin fechas ni descripciones): Lola Nucifora, Cecilia Benedit Debenedetti, Lola de Lusarreta, Olimpia Payer, Consuelo R. González, Paulina Blinder, Dina Mongay, Juana Lumerman Roca, Eloísa J. Dufour, Lucrecia Moyano, Laura Mulhall Girondo, Lía Moyano, Rosa Ferreyra de Roca, Elsa Klappenbach, Leda Ponce de Navarro O'Connor, Katie van Oppen, Helvecia de Z. Wattenhofer, Adela Rabuffi, Elisa Vázquez Cey, Isabel Roca de Larrañaga, María Angélica Keil, Juana Pieri de Puyau, María Pissarro de Pons (se agregan en la edición de 
1981 María Luisa Argento, Sara Cichero, María Esther Bertrand, Anita Payró, Gertrudis Chale); en el apartado sobre escultura, se encuentra a Lola Mora, con imagen y breve relato, y se mencionan a Luisa Isabel Isella de Motteau, Hilda Ainscough, María Carmen de Aráoz Alfaro, Elena F. Guarnaccia Altamira, Ernestina Azlor, María Esther Deretich, Cecilia Marcovich, Augusta Tarnassi de Palma, Noemí Gerstein.

- J. Romero Brest (1951): Ninguna mención. Es notoria, como señala Gluzman (2010), la particular ausencia de nombres femeninos; es evidente que el interés que en la década del '60 tendrían para él figuras de artistas mujeres como Marta Minujín, Delia Cancela, Dalila Puzzovio y Margarita Paksa todavía no se expresaba en los 504.

Ya algunos años antes se advertía una postura a tener en cuenta en la visión de este personaje paradigmático para nuestra historia del arte: en una crítica que Jorge Romero Brest realiza para La Vanguardia (1 de diciembre de 1939) bajo el título general de "Sobre cuatro muestras colectivas", figura, entre otros, el siguiente apartado que funciona como una declaración de principios en cuanto a la relación arte-mujeres: "El Salón Femenino de la Galería Colón no deja de ser una de las habituales reuniones de obras de artistas que buenamente han querido enviarlas.

El menor asomo de selección o de juicio crítico directivo; sin embargo, lo único que podría justificar a salones como éste sería, precisamente, si lo presidiera una idea, un sentimiento, una escuela, una técnica, etc. Aquí no hay nada de todo eso, simplemente obras de mujeres, como si la determinación del sexo tuviera importancia en el acto artístico creador"5. (Giunta et al., 2004: 361-362) - J. E. Payró (1962): En el texto que aparece a modo de introducción, menciona los nombres de Mercedes San Martín de Balcarce, Luisa Sánchez de Arteaga, Procesa Sarmiento, Sor Josefa Díaz y Clucellas y Julia Wernicke (p. 9), pero ninguna de ellas figura en el cuerpo del libro.

- C. Córdova Iturburu (1958): Eugenia Belín Sarmiento, Ana Weiss de Rossi, Mercedes Rodrigué de Soto Acebal, Emilia Bertolé, Blanca R. de Zarlenga, Minerva Daltoe, Yadwiga Alicia Giangrande, Raquel Forner, Norah Borges.

- C. Córdova Iturburu (1978): No tiene mención alguna.

Visiones (¿re-visiones?) posteriores

Un giro se va perfilando con la vuelta a la democracia en los ' 80 , y de a poco comienzan a surgir otras aproximaciones. Para ubicarnos en textos posteriores, que ya hacen (o deberían hacer) eco de nuevas miradas sobre la historiografía del arte, he relevado los siguientes títulos, algunos todavía con el enfoque de "Historia General del Arte en la Argentina", otros con planteos más puntuales o más específicos en cuanto a períodos; ya sea bajo la forma de libros, como de publicaciones que salían a distribución en kioscos de revistas, con llegada a un público más general: - AA. VV. (1982-2005). Historia General del Arte en la Argentina. Buenos Aires:

Academia Nacional de Bellas Artes, 11 tomos.

- García Martínez, J. A. (1985). Arte y enseñanza artística en la Argentina. Buenos Aires: Fundación Banco de Boston. 
- López Anaya, Jorge (2005). Arte Argentino. Cuatro siglos de historia (1600-2000). Buenos Aires: Emecé.

- AA. VV. (2001). Pintura argentina. Panorama del período 1810-2000. Buenos Aires: Ediciones Banco Velox.

- AA. VV. (2010). Museo Nacional de Bellas Artes. Colección. Buenos Aires: Asociación Amigos del MNBA, 2010, 2 volúmenes; versión para su venta en 12 tomos por Grupo Clarín.

Añado aquí los trabajos de Malosetti Costa mencionados al inicio, por considerarlos pioneros en cuanto a nuestro interés: - Malosetti Costa, Laura (2000). "Una historia de fantasmas. Artistas plásticas de la generación del ochenta en Buenos Aires". En VI Jornadas de Historia de las Mujeres y I Congreso Iberoamericano de Estudios de las Mujeres y de Género, Voces en conflicto, espacios en disputa, Buenos Aires, FFyL-UBA (CDROM).

- Malosetti Costa, Laura (2001). Los primeros modernos. Arte y Sociedad en Buenos Aires a fines del Siglo XIX. Buenos Aires: Fondo de Cultura Económica.

En estas publicaciones podemos encontrar nombres repetidos, algunos omitidos, y nuevas menciones en algunos casos; señalaremos aquellos que se encuentran en el marco que compete a nuestro análisis: - AA. VV., Historia General del Arte en la Argentina (1982-2005): Tomo 3: Siglo xix hasta 1876 (1984). En "La Pintura”, de Adolfo Luis Ribera, se nombran a Antonia Brunet de Annat y a Andrea Bacle, con descripción y reproducciones de la primera; luego a Josefa Díaz y Clucellas, Procesa Sarmiento de Lenoir, Eugenia Belín Sarmiento.

En "El grabado y la litografía”, de Bonifacio del Carril, aparece nuevamente Andrea Bacle.

Tomo 6: Fines del siglo xix y comienzos del xx (1988). En "La Pintura", de Julio E. Payró, se mencionan a Procesa Sarmiento de Lenoir, Josefa Díaz y Clucellas, y se refiere a la participación de las "niñas de la sociedad", pues "crece en ese momento el número de mujeres artistas de la Argentina" (p. 151), que se dan a conocer en los Salones del Ateneo: Matilde Villaret, Ana Galán, Elena Somellera, Eugenia Belín Sarmiento, Victoria Aguirre, Sofía Posadas, María Obligado de Soto y Calvo, Elina González Acha, Julia Wernicke; luego, dentro de los participantes del Salón Nacional, aparecen Lía Gismondi, Catalina Mórtola de Bianchi, Ana Weiss de Rossi. Del mismo autor, en "La Escultura” figura muy brevemente Lola Mora, con imagen de su fuente de Las Nereidas.

Tomo 7: Comienzos del siglo xx (1995). En "Fotografía argentina", de Sara Facio, aparece Annemarie Heinrich (junto con Grete Stern), con reproducción de algunas de sus obras. En "El grabado en la Argentina", de Nelly Perazzo, se menciona el nombre de Catalina Mórtola de Bianchi, y de Norah Borges se escribe un breve párrafo, también se nombran a Margarita Portela Lagos, María Esther Leanes, Elba Villafañe, María Carmen Aráoz Alfaro, María Catalina Otero Lamas, Hemilce Saforcada, Nélida Demichelis, Manuela Alles Monasterio.

Tomo 8: Comienzos del siglo XX (1999). En "La pintura en la Argentina”, de Nelly Perazzo, figuran Norah Borges y Raquel Forner, con muy breve alusión y una reproducción de cada una. Más adelante se mencionan a Olimpia Payer y Gertrudis Chale.

- J. A. García Martínez (1985): Andrea Paulina Macaire de Bacle, Antonia Brunet de Annat, Mercedes de Anchorena de Aguirre, Procesa Sarmiento de Lenoir, Bienvenida Sarmiento, Eugenia Belín Sarmiento, Lucila 
Antepara de Godoy, Juana Manso, Daría Echagüe, Catalina Mórtola de Bianchi, Sor Josefa Díaz y Clucellas, Ana Galán de Coll, Rosario Pujato de Crespo, Raquel Forner, Lucía Capdepont; a todas se refiere, en general, de forma concisa.

- J. López Anaya (1997): Del siglo xix, Lola Mora, Andrea Bacle; del siglo xx, Norah Borges, Raquel Forner.

- J. López Anaya (2005): En este libro, cuyo enfoque está planteado desde su Preliminar "con la inclusión de problemas de carácter más general" (p. 12), el autor parece hacerse eco de Pagano y de revisiones bibliográficas más cercanas, y la mención y dedicación a artistas mujeres se amplía con respecto a su texto anterior; es así que aparecen Andrea Bacle, Antonia Brunet de Annat, Sor Josefa Díaz y Clucellas, Procesa Sarmiento de Lenoir, Luisa Sánchez de Arteaga, Julia Wernicke, Eugenia Belín Sarmiento, Sofía Posadas, Diana Cid García, Allardice Graham de Witt, Emilia Bertolé, Catalina Mórtola de Bianchi, Lola Mora, Luisa Isabel Isella, Elina González Acha de Correa Morales, María Obligado de Soto y Calvo, Hortencia Berdier, Norah Borges, Raquel Forner, Elena Cid, Gertrudis Chale, Maruja Mallo.

- L. Malosetti Costa (2000): Dedica el trabajo a los casos de Sofía Posadas y Diana Cid García (con análisis extendido); también menciona a María Huergo y a Allardice Graham de Witt.

- L. Malosetti Costa (2001): en el capítulo ix, "Pintores y poetas II. El Ateneo”, aparecen Eugenia Belín Sarmiento, Sofía Posadas, Diana Cid García; en el capítulo x, "Schiaffino, Darío y el proyecto modernista", Diana Cid García, Allardice Graham de Witt, Sofía Posadas; en ambos capítulos, además de las articulaciones con la trama de su tiempo, cita artículos periodísticos que comentan la participación de las "damas" y las "señoritas" en los Salones del Ateneo.6 - AA. VV. (2001). Pintura argentina. Panorama del período 1810-2000 (2010):

Fascículo 7: Precursores II. En "Retratos y escenas costumbristas", de Laura Malosetti Costa, se dedica una parte del texto a Procesa Sarmiento de Lenoir y a Sor Josefa Díaz y Clucellas.

Fascículo 9: Identidad y vanguardia. En "Tensiones de la Modernidad”, de Diana Wechsler, aparecen Raquel Forner y Gertrudis Chale.

Fascículo 11: Primeras vanguardias. En "El estallido de los lenguajes", de Sylvia Iparraguirre, figura Forner. En "Modernidad y vanguardia en la década del 20", de Patricia Artundo, nuevamente nos encontramos con Forner.

- AA. VV., Museo Nacional de Bellas Artes. Colección (2010): dentro del período que nos atañe se mencionan y analizan obras de Emilia Bertolé, Gertrudis Chale y Raquel Forner.

No se realiza ninguna otra alusión a la escasa presencia de mujeres en el patrimonio del Museo (que refleja en esto claramente las pautas de la gestión de Schiaffino); asimismo en la disposición más reciente de las salas, la selección de obras en lo que se refiere al siglo xix tampoco revela una revisión al respecto. Gracias a la información otorgada por el Departamento de Documentación y Registro, sabemos que, además de las mencionadas, el patrimonio del MNBA cuenta con obras de Julia Wernicke, Leonor Terry Quirno Costa, Ana Weiss de Rossi, Elena Ruiz, Elina González Acha de Correa Morales, Lía Correa Morales de Yrurtia, Lía Gismondi, Rosalía Soneira, Norah Borges. En cuanto a las imágenes, muchas de estas omisiones en los tomos de Colección están salvadas en el sitio web del museo: http://www.mnba.gob.ar/coleccion/arte-argentino. 
Como podemos observar, en el segundo bloque del corpus bibliográfico ya no se soslaya la presencia de las mujeres: las menciones de muchos nombres continúan, y, aunque otros dejan de figurar, nuevos nombres aparecen. Pero, si bien en algunos casos se da un espacio dedicado a las referencias sobre su vida y obra, no ha habido en general importantes reconsideraciones que hayan planteado una mirada renovadora con respecto a la crítica historiográfica. En el caso de los enfoques más abarcadores, continúa sin aparecer un debate profundo sobre las circunstancias que vivenciaron las "primeras modernas" y aquellas que las siguieron; no se estudian las condiciones de producción, de circulación, la recepción. Dentro de estas estimaciones se incluirían los estudios sobre las poéticas: si había diferencias entre los géneros artísticos en los que las mujeres incursionaban y los desarrollados por sus colegas varones; si dentro del mismo género existían resoluciones diferentes según fueran planteadas por mujeres o por varones. Todo ello sin suponer, por supuesto, que se puedan reconocer marcas "femeninas", al estilo de nuestros primeros historiadores, sino pensando las obras justamente como productos del entramado de esas condiciones mencionadas, dentro de las cuales no debe olvidarse el nivel correspondiente a la formación: las posibilidades de estudio, el acceso a él, el currículo.

Quizás se considera que estos análisis quedan fuera de las historias generales, y que deben ser llevados a cabo por estudios más específicos, como los de Malosetti Costa, o los más recientes de Gluzman. Claro que en la actualidad ya no buscamos la perspectiva de "una" historia general del arte (aunque de todos modos estos registros siguen siendo útiles a quienes se están formando en la disciplina), sino que hablamos de "historias" del arte7, y es a partir de este lugar que esperamos sigan saliendo a la luz los nuevos acercamientos que generaciones más recientes de historiadoras e historiadores del arte están llevando a cabo.

Apelo también a que se conozcan planteos similares de otros países latinoamericanos.

Por otra parte, si avanzamos hacia la década de 1940 y seguimos hasta la actualidad, sabemos que la cantidad de artistas mujeres se multiplica, podríamos decir, de manera exponencial, y se suman agentes como historiadoras del arte, críticas, curadoras, galeristas, coleccionistas, gestoras, directoras de museos y de centros culturales: hechos reflejados en los diversos registros escritos que sustentan el quehacer en el campo del arte, y cuyo estudio son tema para otros muchos abordajes que investigadoras e investigadores también, y diría, al fin, están elaborando con compromiso y seriedad.

Notas

1. El presente artículo es uno de los trabajos derivados del proyecto de investigación "Artistas argentinas entre 1911 y 1940. Nuevas estrategias discursivas sobre el campo artístico" (2010-2013), inscripto dentro del Programa de Reconocimiento Institucional (PRI) de Equipos de Investigación de la Facultad de Filosofía y Letras, de la Universidad de Buenos Aires, y radicado en el Instituto Interdisciplinario de Estudios de Género de dicha Facultad.

El grupo de estudio e investigación del mencionado proyecto incluyó a Micaela Fernández Darriba (Comunicación Social, UBA), Georgina Gluzman (Artes, UBA; CONICET), Ana Hib (Artes, UBA), María Laura Rosa (Historia del Arte, U. Complutense de Madrid; IIEGE, UBA) y Malena San Juan (Artes, UBA), con el objetivo de reflexionar y problematizar la escritura de la historia del arte argentino y latinoamericano, así como también debatir sobre las construcciones de lo femenino en el campo de las representaciones visuales. Dentro de las tareas llevadas a cabo, el Archivo José León Pagano (Museo de Arte Moderno de Buenos Aires) y el 
Archivo Witcomb (Fundación Espigas) fueron consultados en su totalidad; también se estudió el Archivo Pío Collivadino (Universidad Nacional de San Martín).

2. Como prueba de esta importante participación, podemos mencionar el álbum Academia Nacional de Bellas Artes y Escuela de Artes Decorativas e Industriales 1878-1928. Buenos Aires-República Argentina, hallado y registrado fotográficamente en la Biblioteca Nacional por G. Gluzman. Luego de su relevamiento, pude observar que figuran los nombres de mujeres pertenecientes al cuerpo docente y administrativo, fotografías de alumnas de los distintos cursos, fotografías de obras de las alumnas, y listados de expositores premiados, de expositores en el Salón alumnos de la Academia y de profesores de dibujo egresados de la Academia donde también se encuentran nombradas mujeres. Como observaciones generales podemos decir que en el registro fotográfico de todos los cursos se hallan gran cantidad de alumnas, en pie de igualdad con los varones, también se reproducen muchas obras de ellas, y en los listados se ven porcentajes muy ilustrativos de una progresión que indica una notoria participación de las mujeres en los ámbitos de formación y una merma importante en los ámbitos de reconocimiento: en la lista de Profesores de Dibujo egresados de la Academia, de 615 nombres, 385 son de mujeres y 230 de varones; en "Expositores en el Salón de Bellas Artes también alumnos de la Academia", de 127 nombres 30 son de mujeres, mientras que en "Expositores premiados en el Salón de Bellas Artes que fueron alumnos de la Academia", de 49 nombres 6 son de mujeres. En otros artículos de publicaciones periódicas sobre la Academia hallados en el archivo de Pío Collivadino vemos, tanto en las fotografías como en los relatos, nuevamente la gran presencia de mujeres que cursan estudios diversos sobre artes plásticas o decorativas. Por otro lado, tanto del Archivo José León Pagano como del de Witcomb, se desprende la notoria presencia de mujeres en los Salones Nacionales.

3. En retrospectiva, este "apartado" de Pagano parecería funcionar como el antecedente de una tendencia que se observa a partir de la recuperación de la democracia: el armado de exposiciones colectivas de artistas mujeres que se llevaron a cabo con diversos criterios y justificaciones, y con catálogos o al menos algún material impreso de registro. En el entonces Centro Cultural Ciudad de Buenos Aires se presentaron varias, desde mediados de los '80: se inauguró esta línea con el ciclo de "Mitominas" ("Mitominas. Un paseo a través de los mitos", noviembre de 1986, "Mitominas 2. Los mitos de la sangre”, noviembre de 1988; véanse diversos artículos de María Laura Rosa, esp. "El despertar de la conciencia. Impacto de las teorías feministas sobre las artistas de Buenos Aires durante las décadas del '70 y ' 80 ”, Artelogie, 5, octubre 2013 [http://cral.in2p3.fr/artelogie/spip.php?article247]); de esta década también es La mujer en la plástica argentina I, Centro Cultural las Malvinas, Buenos Aires, 1988. En los 90 encontramos diversas muestras colectivas: en el primer centro cultural mencionado, ya con el nombre actual de Centro Cultural Recoleta: "Tributo a Frida", marzo de 1996; "Juego de Damas," noviembre de 1996 y "Mujeres", agosto de 1998; en el Museo Nacional de Bellas Artes, junto con el Consorci de Museus de la Comunitat Valenciana, se exhibe "Femenino Plural. Arte de mujeres al borde del tercer milenio", marzoabril de 1998; por su parte, el Centro Cultural Borges presenta "Tajos Bajos", abril de 1997 (véanse varios trabajos de Natalia Pineau, esp. "El género en cuestión: tres exposiciones de artistas mujeres durante los años '90 en Buenos Aires”, en Herrera, María José (dir.), Exposiciones de arte argentino y latinoamericano: curaduría, diseño y políticas culturales, Córdoba-Buenos Aires, Escuela Superior de Bellas Artes Dr. Figueroa Alcorta y Grupo de Estudios sobre Museos y Exposiciones, 2011), y más tarde “Autorretrato. 130 artistas mujeres/ 24 curadoras", marzo de 2001; un poco más adelante tenemos "Mujeres Argentinas. 
En torno al cuerpo, el territorio y la manualidad", con tres itinerancias (Mar del Plata, Río Gallegos, Buenos Aires) en 2003. Existen varios otros casos, en galerías o promovidos por alguna empresa o fundación, o por el mismo Gobierno de la Ciudad de Buenos Aires, y muchos presentados en cercanía a fechas alusivas, como el 8 de marzo, Día de la Mujer.

4. Sin embargo, por otra parte encontramos en el Archivo Jorge Romero Brest (FFy L, UBA) material de textos escritos por el crítico para catálogos y notas de periódicos y revistas sobre Raquel Forner. Asimismo, como Director del Museo Nacional de Bellas Artes, prologó el catálogo y organizó la exposición Raquel Forner; pinturas series de las lunas, 1958-1962, en 1962, un año antes de la finalización de su gestión.

5. El subrayado es nuestro.

6. Es de hacer notar que M. Costa también se ha ocupado de incorporar obras de artistas mujeres en el relato de las curadurías de sus exhibiciones, como en "Primeros modernos en Buenos Aires. Del viaje a Europa a las exposiciones del Ateneo (1876-1896)", Museo Nacional de Bellas Artes, 2007, o en la reciente "Yo, nosotros, el arte", Fundación OSDE, 2014.

7. Al respecto, véanse Baldasarre, María Isabel y Silvia Dolinko (eds.), Travesías de la imagen. Historias de las Artes Visuales en la Argentina, Buenos Aires, CAIA-EDUNTREF, vol. 1, 2011, vol. 2, 2012; y el ya mencionado volumen dirigido por María José Herrera, Exposiciones de arte argentino y latinoamericano..., donde encontramos trabajos de Pineau y de Rosa.

Bibliografía citada

Giunta, A. et al. (comps.) (2004). Jorge Romero Brest. Escritos I (1928-1939). Buenos Aires: Instituto de Teoría e Historia del Arte "Julio E. Payró", FFyL, UBA.

Gluzman, G. (2010). “Omisiones y presencias veladas: artistas argentinas en las historias del arte”. En Actas del II Congreso Feminista Internacional Argentina. 100 años de Historia Social y de Género de las Mujeres (CDROM).

Malosetti Costa, L. (2000). “Una historia de fantasmas. Artistas plásticas de la generación del ochenta en Buenos Aires". En VI Jornadas de Historia de las Mujeres y I Congreso Iberoamericano de Estudios de las Mujeres y de Género, Voces en conflicto, espacios en disputa. Buenos Aires: FFyL, UBA.

Malosetti Costa, L. (2001). Los primeros modernos. Arte y Sociedad en Buenos Aires a fines del Siglo XIX. Buenos Aires: Fondo de Cultura Económica.

Abstract:

The article aims to trace a path of the mentions of those artists who pass through our art history from its beginnings to the late 1930s, making a comparison of the texts closest in time to review changes and continuities. In our early days, women are taught, they create and appear in journalistic reviews; but recognition of the canonical texts or the awards does not arrive or are few. From the 1940s until the present, the quantity of 
women artists increases. In addition, agents such as art historians, critics, curators, gallery owners, collectors, managers, directors of museums and cultural centers are included.

Key words:

argentine art - women artists - art historiography - canon.

Resumo:

O texto tem como objetivo fazer um mapa das menções das artistas que atravessam nossa historiografia da arte seus começos até fins de 1930, comparando textos mais pertos no tempo para revisar mudanças e permanências. No início, as mulheres se instruem, criam e aparecem em críticas jornalísticas, mas o reconhecimento dos textos canônicos ou dos prêmios não chegaram ou estavam de modo reduzido. A partir da década de 1940 e até hoje, a quantidade e o registro das artistas mulheres se multiplica, e sumam-se agentes como historiadoras da arte, críticas, curadoras, galeristas, colecionadoras, gestoras, diretoras de museus e de centros culturais.

Palavras chave:

arte argentino - artistas mulheres - historiografia da arte - cânon.

Repertorio de artistas mujeres en la historiografía canónica del arte argentino: un panorama de encuentros y desencuentros fue publicado de la página 49 a página58 en Cuadernos del Centro de Estudios de Diseño y

Comunicación $N^{\circ} 60$ 